\title{
Revealing Atomic-Scale Surface Segregation, Intermixing and Internal Ordering in Fuel Cell Nanocatalysts by Aberration-Corrected Spectroscopic Imaging
}

\author{
Huolin L. Xin, Deli Wang, ${ }^{* *}$ Hector D. Abruña, ${ }^{* *}$ David A. Muller, ${ }^{* * *}$ \\ * Department of Physics, Cornell University, Ithaca, NY 14853 \\ ** Department of Chemistry and Chemical Biology, Cornell University, Ithaca, NY 14853 \\ *** School of Applied and Engineering Physics \& Kavli Institute, Cornell Univ., Ithaca, NY 14853
}

To achieve the optimum catalytic activity for fuel cell nanocatalysts, the ability to control the surface composition with atomic precision is required. Using aberration-corrected electron microscopy and EELS [1], we can now substantially improve our understanding of the surface/sub-surface chemistry of nanocatalyst particles [2]. Here, we demonstrate a direct, atomic-resolution visualization and a precise determination of surface segregation, sub-surface intermixing, and internal ordering-of each species of atoms - in fuel cell nanocatalysts and study the alloying trends across the $3 d$ series with Pt and Pd. Many previous studies of fuel cell nanocatalysts have relied on the atomic number sensitivity of HAADF-STEM images to unscramble the surface composition of nanoparticles [3]. For example in Figure 1c, a $\mathrm{Pt}_{3} \mathrm{Co}$ nanoparticle was imaged by an aberration-corrected ADF-STEM $\left(100 \mathrm{keV}, \alpha_{\max } \approx 30 \mathrm{mrad}\right.$ ) along the [001] zone axis. The qualitative match to multislice simulation of a modeled Pt-Co nanoparticle (Fig. 1a and 1b) indicates the bulk of the particle is $\mathrm{L}_{2}$ ordered and the first 2-3 atomic layers on the $\{100\}$ facets - as indicated by the red arrows-appear free of the ordered super-lattice structure. The simulation suggests the shell is likely to be pure Pt planes rather than a Pt-Co alloy. However, this analysis relies upon a-priori knowledge of the particle shape and the lattice structure. Additional factors, such as strain fields and metal oxide clusters decorated on surfaces, can make the analysis elusive, especially for elements with similar atomic numbers.

However, much of the ambiguity can be resolved by atomic-resolution EELS mapping. A selection of our mapping results of $\mathrm{Pt}_{3} \mathrm{Fe}$ and $\mathrm{Pt}_{3} \mathrm{Cr}$ nanocatalysts are shown in Figure $2 \mathrm{a}$ and $2 \mathrm{~b}$. The chemical maps obtained are striking. The $\mathrm{L}_{2}$ ordering of $\mathrm{Fe}$ and $\mathrm{Cr}$ is directly visualized. Because the elastic scattering intensity is dominated by $\mathrm{Pt}$, we can use the simultaneously acquired ADF image as an approximate map of Pt to determine the Pt segregation thickness. We found that 2-3 atomic layers of segregated $\mathrm{Pt}$ is nearly universal on every facet of the particles imaged. However, despite the predominant $\mathrm{Pt} / \mathrm{Pt}_{3} \mathrm{M}$ core-shell structure, the surfaces of these particles are decorated with the oxides of the $3 d$ alloying metals. These metal oxide cluster can obscure any attempts of using HAADF-STEM images to uncover the surface composition. It is also interesting to note that, in Fig. $2 b_{3}$, a single column of $\mathrm{Cr}$ is present one super-lattice spacing away from its nearest-neighbor $\mathrm{Cr}$ column but not closer or further. Moreover, Fig. 2c shows the mapping of a trimetallic $\mathrm{Pd}-\mathrm{Cu}-\mathrm{Co}$ alloy particle. Because $\mathrm{Co}$ and $\mathrm{Cu}$ are very close in atomic number, it is almost impossible to distinguish the distribution of the two based on a HAADF-STEM image. Our EELS maps reveal that the Co stays $\sim 1 \mathrm{~nm}$ away from the surface of particle, whereas $\mathrm{Cu}$-alloyed with $\mathrm{Pd}$ - extends nearly to the outer surface. These precise atomic-scale measurements are crucial for understanding of the effect of our synthesis parameters such as annealing temperature and ambient conditions. Thus, the atomic-scale spectroscopic imaging technique presented here is critical for the discovery of new catalysts with optimized activities [4].

References

[1] D. A. Muller et al., Science 319, 1073 (2008).

[2] H. Xin et al., Microscopy and Microanalysis 16, 104 (2010).

[3] S. Chen et al., J Phys Chem C 113, 1109 (2009).

[4] Funded by the Energy Materials Center at Cornell, an Energy Frontier Research Center (DOE \#DE-SC0001086). 


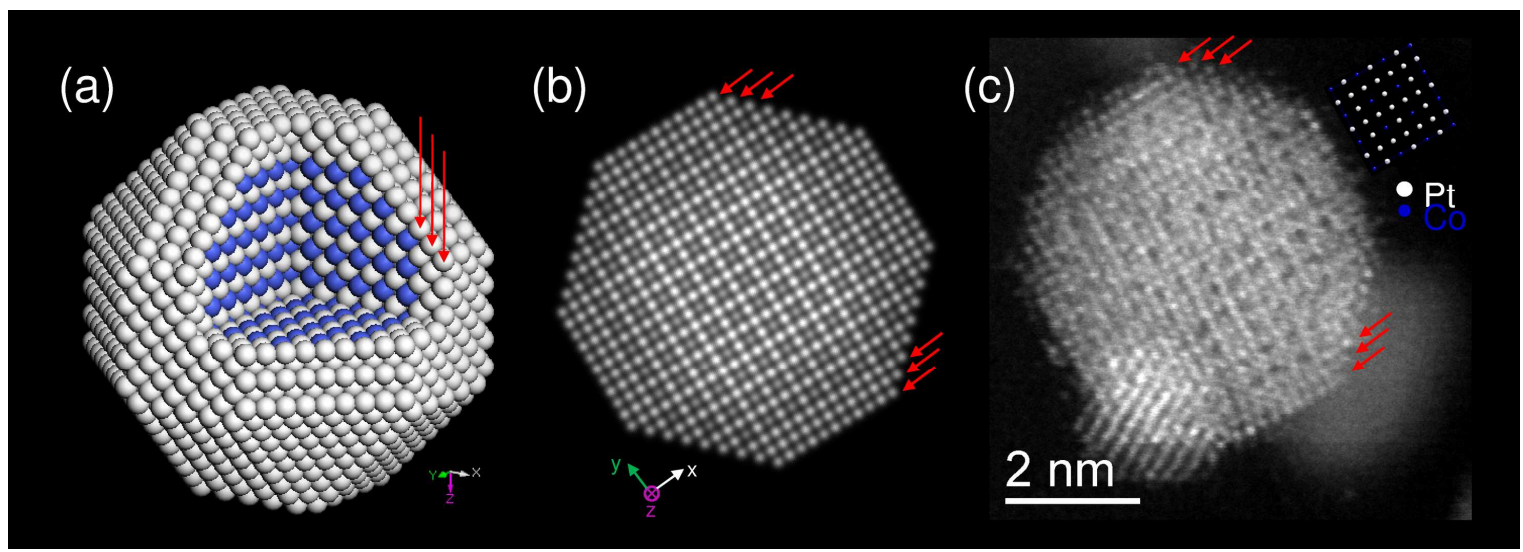

Fig. 1. (a) The reconstructed atomic structure of the $\mathrm{Pt}_{3} \mathrm{Co}$ core-shell nanoparticle. The distance between the three major facets $(\{111\},\{001\},\{110\})$ were chosen to match the morphology of the particle shown in (c) rather than using a Wulff construction. (b) The simulated ADF-STEM image using a multislice code. (c) The experimental atomic-resolution ADF-STEM image after Richardson-Lucy deconvolution (4 iterations).

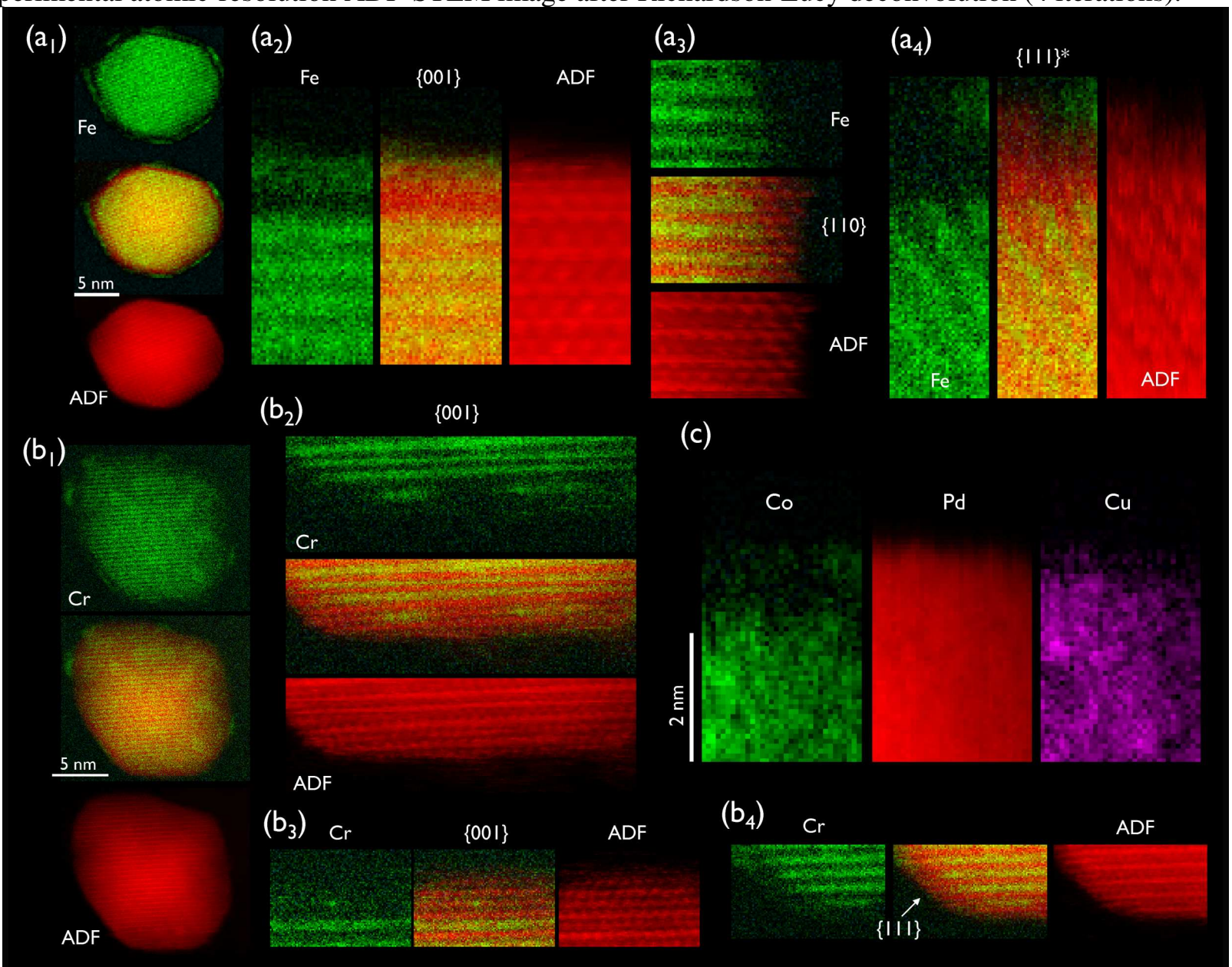

Fig. 2. Atomic-resolution EELS mapping of surface segregation, sub-surface intermixing and internal ordering of fuel cell nanocatalyst particles. (a) Atomic-scale EELS mapping of a $\mathrm{Pt}_{3} \mathrm{Fe} \mathrm{L}_{2}$ ordered nanoparticle: mapping of $\left(a_{1}\right)$ the whole particle, $\left(a_{2}\right)$ the $(001)$ facet, $\left(a_{3}\right)$ the $(-110)$ facet and $\left(a_{4}\right)$ the $(1-11)$ facet. (b) EELS mapping of a $\mathrm{Pt}_{3} \mathrm{Cr} \mathrm{L} 1_{2}$ ordered particle: atomic-resolution mapping of $\left(\mathrm{b}_{1}\right)$ the whole particle, $\left(b_{2}\right)$ the $(00-1)$ facet, $\left(b_{3}\right)$ the $(001)$ facet and $\left(b_{4}\right)$ the (1-1-1) facet. $(c)$ Atomic-scale EELS mapping of a selected surface area of a 10-nm Pd-Cu-Co particle. 